\title{
Comparación de modelos de propagación de ondas de radio de un canal inalámbrico en un entorno urbano de la ciudad de Barranquilla
}

\section{Comparison of radio wave propagations models of a wireless channel in the urban environment of the city of Barranquilla}

\author{
DOI: https://doi.org/10.17981/cesta.02.01.2021.03
}

Artículo de investigación científica. Fecha de recepción: 10/02/21. Fecha de aceptación: 25/06/21.

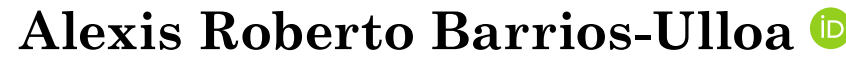 \\ Universidad de Sucre. Sincelejo (Colombia) \\ alexis.barrios@unisucre.edu.co
}

Para citar este artículo:

A. Barrios-Ulloa, "Comparación de modelos de propagación de ondas de radio de un canal inalámbrico en un entorno urbano de la ciudad de Barranquilla”, J. Comput. Electron. Sci.: Theory Appl., vol. 2, no. 1, pp. 31-38, 2021. https://doi.org/10.17981/cesta.02.01.2021.03

Resumen-Los modelos de propagación empíricos son ampliamente usados para calcular las pérdidas por trayectoria en un canal inalámbrico en diferentes tipos de escenarios, y sus resultados son tenidos en cuenta al momento de seleccionar la ubicación de estaciones base y planificar su área de cobertura. Los modelos Walfisch-Ikegami, Interino de la Universidad de Stanford (SUI) y COST-231 Hata fueron evaluados en este trabajo con el propósito de estimar su efectividad. La potencia en un receptor operando en la banda de $1900 \mathrm{MHz}$ fue medida en diferentes ubicaciones de una zona urbana de Barranquilla, Colombia, y los datos obtenidos se utilizaron en la comparación. La efectividad de la predicción de pérdidas por parte de los modelos fue analizada a través del cálculo del error relativo y error de predicción, mostrando que el Walfisch-Ikegami presentó menor error relativo en comparación con el SUI tipo B y el COST-231 Hata. Los valores de error obtenidos fueron altos, lo cual indica que los modelos evaluados no predicen adecuadamente las pérdidas medidas en el escenario considerado.

Palabras clave- Error de predicción; error relativo; entorno urbano; modelo de propagación empírico; pérdida por trayectoria

\begin{abstract}
Empirical propagation models are widely used to calculate path losses in a wireless channel in different types of scenarios, and their results are considered when selecting the location of base stations and planning their coverage area. The Walfisch-Ikegami, Stanford University Interim (SUI) and COST-231 Hata models were evaluated in this work in order to estimate their effectiveness. The power in a receiver operating in the $1900 \mathrm{MHz}$ band was measured in different locations in an urban area of Barranquilla, Colombia, and the data obtained was used in the comparison. The effectiveness of the loss prediction by the models was analyzed through the calculation of the relative error and prediction error, showing that the Walfisch-Ikegami presented the lowest relative error compared to the SUI type B and the COST-231 Hata. The error values obtained were high, which indicates that the evaluated models do not correctly predict the losses measured in the considered scenario.
\end{abstract}

Keywords- Empirical propagation model; prediction error; relative error; trajectory loss; urban setting. 


\section{INTRODUCCIÓN}

En un sistema de comunicación inalámbrico sin línea de vista (NLOS) se introducen múltiples trayectorias de la señal de radio, afectando el valor de la potencia a la entrada del receptor. En las redes inalámbricas el canal de propagación de radio es muy importante y su modelamiento es un factor crítico y complejo [1], [2]; y es por esto que el cálculo de la pérdida de propagación resulta ser uno de los parámetros mas importantes en el dimensionamiento de redes celulares [3]. Por consiguiente, el canal de radio requiere de un análisis detallado dada su importancia en el diseño de sistemas de comunicaciones [4].

Los modelos de propagación son ampliamente usados en la caracterización de la atenuación de la señal de radio en un enlace inalámbrico. Se definen como la diferencia entre potencia efectiva transmitida y la potencia recibida [1]. Posteriormente, sus resultados se emplean en la predicción matemática de radiofrecuencia entre el transmisor y el área de cobertura objetivo [5].

Los parámetros generalmente considerados al momento de caracterizar la propagación en un canal inalámbrico son la frecuencia y la distancia entre transmisor y receptor. No obstante, también se incorporan otros, dentro de los cuales están: altura de antenas, tipo de entorno, características de los obstáculos. Estos modelos resultan de gran importancia en la predicción de áreas de cobertura, en análisis de interferencia y para determinar parámetros de Estaciones Base (EB) [6].

Existen diferentes categorías de modelos de propagación, por ejemplo en [1] los clasifican como teóricos y empíricos mientras que [7] los dividen en empíricos, determinísticos y semideterminísticos. De estas categorías, los empíricos son una opción práctica y eficiente de calcular la pérdida de potencia en un canal inalámbrico a partir de mediciones de campo. Sin embargo, su principal desventaja es la falta de precisión que se presentan al ser usados en entornos distintos de aquellos en los cuales fueron concebidos [8].

Barranquilla es una de las ciudades mas grandes de Colombia y una de las más desarrolladas. En ella se dan diferentes usos de suelo para la construcción, presentándose así diferentes entornos de propagación de acuerdo con la recomendación hecha por la Unión Internacional de Telecomunicaciones [9]. En esta ciudad se seleccionó un escenario de tipo urbano donde se llevó a cabo un estudio comparativo que incluyó tres modelos de propagación: COST 231-Hata, Walfisch-Ikegami e Interino de la Universidad de Stanford (SUI). El objetivo es valorar la efectividad de estos modelos en el escenario escogido, a partir de mediciones de campo.

El artículo presenta la siguiente estructura, en la cual la sección II muestra una descripción de investigaciones anteriores relacionadas con este trabajo, para continuar con la sección III donde se explican los modelos seleccionados en esta comparación y, además, el procedimiento de medición. En la siguiente sección IV se muestran los resultados; para en la sección V presentar las conclusiones y, finalmente en la sección VI entregar las recomendaciones necesarias para trabajos futuros.

\section{Trabajos Relacionados}

Debido a que los modelos empíricos tienden a presentar cambios en las estimaciones de pérdida potencia cuando son usados en entornos diferentes de aquellos donde fueron creados, se han desarrollado diferentes estudios comparativos para determinar la efectividad de diferentes modelos a diferentes frecuencias y en distintos escenarios.

En el 2016 se compararon diferentes modelos de propagación utilizando el simulador NS-3 para una red LTE en un terreno caracterizado como suburbano y, además, los categorizaron de acuerdo con la complejidad computacional [10]. El mismo año realizaron una comparación entre los modelos Walfisch-Ikegami, SUI y Ericsson en las bandas de $850 \mathrm{MHz}$ y $1800 \mathrm{MHz}$ [11]. En otras investigaciones determinaron las características de propagación de escenarios urbanos y rurales de Shanghai en once bandas de radiofrecuencia entre los $700 \mathrm{MHz}$ y los $3500 \mathrm{MHz}$, a través de la comparación de los modelos COST-231 Hata y Okumura-Hata [12]. Además, ciertos estudios compararon los modelos de pérdidas en el espacio libre, COST-Hata y Walfisch-Ikegami, con el objetivo de valorar su efectividad en un entorno urbano en la banda de $1800 \mathrm{MHz}$ en Ankara, Turquía [13]. En ese mismo estudio [13], comprobaron cinco modelos de propagación en la banda de $1800 \mathrm{MHz}$ para determinar cual resulta ser el más adecuado para el dimensionamiento de redes celulares de cuarta generación (4G).

Los ejemplos de trabajos relacionados, aquí presentados demuestran que la comparación de modelos de propagación resulta ser relevante en la investigación sobre caracterización de la atenuación en escenarios donde se desplieguen redes inalámbricas, entre las que se encuentran los sistemas de telefonía móvil, también conocidos como celulares. 


\section{Materiales y MÉTodos}

Para realizar la comparación, tres modelos de propagación fueron seleccionados de acuerdo con sus características y también a partir de las condiciones de topografía, alturas de antenas, banda de frecuencia y tipo de celda que se configuraron durante la toma de datos.

\section{A. Modelo Cost 231-Hata}

El COST-231 Hata se deriva de un modelo propuesto anteriormente por Hata. Fue concebido principalmente para usarse en macroceldas y en bandas de frecuencias superiores a $1500 \mathrm{MHz}$. Además, es válido en antenas de transmisión ubicadas entre $30 \mathrm{~m}$ y $200 \mathrm{~m}$, y de $1 \mathrm{~m}$ a $10 \mathrm{~m}$ en la antena del receptor [14]. La Ecuación (1) permite calcular las pérdidas por trayecto con el modelo COST-231 Hata:

$$
\begin{aligned}
& P L(d B)=46.3+33.9 \log _{10} f-13.82 \log _{10} h_{b} \\
& -a h_{m}+\left(44.9-6.55 \log _{10} h_{b}\right) \log _{10} d+C_{m}
\end{aligned}
$$

Siendo $f$ la frecuencia expresada en $M H z, d$ la distancia del transmisor al receptor dada en $K m s, h_{\mathrm{b}}$ es la altura de la antena de EB en metros, y $a h_{m}$ es un factor de corrección de la antena de la Estación Móvil (EM), que depende de la densidad de las estructuras en el entorno urbano, caso en el cual se calcula mediante (2):

$$
a h_{m}=\left(1.11 \log _{10} f-0.7\right) h_{r}-\left(1.56 \log _{10} f-0.8\right)
$$

En esta ecuación $h_{r}$ corresponde a la altura de la antena de la EM en metros y $\mathrm{Cm}$ toma valores de $3 \mathrm{~dB}$ en entornos urbanos.

\section{B. Modelo Walfisch -Ikegami}

El modelo Walfisch-Ikegami se desarrolló a partir de una descripción estadística precisa de un entorno urbano [15]. Es válido con alturas de la EM de entre $1 \mathrm{~m}$ y $3 \mathrm{~m}$ y de la antena de EB entre $4 \mathrm{~m}$ y $50 \mathrm{~m}$ de altura [16]. Además, se considera para un rango de de $800 \mathrm{MHz}$ a $2000 \mathrm{MHz}$. Walfisch-Ikegami contempla enlaces NLOS y con línea de vista (LOS). En (3) y (4) se presentan las ecuaciones que definen este modelo:

$$
\begin{aligned}
& P L(d B)=L_{o}+L_{r t s}+L_{m s d} \text { para NLOS } \\
& P L(d B)=L_{o} \text { para LOS }
\end{aligned}
$$

$L_{0}$ corresponde a pérdidas en el espacio libre calculadas mediante (5):

$$
L_{o}=32.4+20 \log _{10} d+20 \log _{10} f
$$

En la que $d$ corresponde a la distancia en $K m s$ que hay de la EB a la EM, y $f$ es el valor de frecuencia del enlace en $M H z$. $L_{\mathrm{rts}}$ son pérdidas por difracción azotea-calle y pérdidas por dispersión, y se presentan en (6):

$$
\begin{aligned}
L_{r t s}= & -16.9-10 \log _{10} w+10 \log _{10} f \\
& +20 \log _{10}\left(\Delta h_{\text {mobile }}\right)+L_{\text {ori }}
\end{aligned}
$$

Siendo $w$ la anchura de las calles, $\Delta h_{\text {mobile }}$ es la diferencia entre altura promedio de construcciones que se encuentra alrededor de la EB y la altura de la antena de EM. $L_{\text {ori }}$ es una función de orientación de la antena con respecto a la calle, que está dada por (7):

$$
L_{\text {ori }}=\left\{\begin{array}{cc}
-10+0.354 \varnothing & \text { para } 0^{\circ} \leq \varnothing<35^{\circ} \\
2.5+0.075(\varnothing-35) & \text { para } 35^{\circ} \leq \varnothing<55^{\circ} \\
4.0-0.114(\varnothing-55) & \text { para } 55^{\circ} \leq \varnothing \leq 90^{\circ}
\end{array}\right.
$$


$L_{\text {msd }}$ representa la pérdida por difracción debido a múltiples obstáculos y se especifica mediante (8):

$$
L_{m s d}=L_{b s h}+K_{a}+K_{d} \log _{10} d+k_{f} \log _{10} f-9 \log _{10} b
$$

Donde $L_{\mathrm{bsh}}$ y $K_{\mathrm{a}}$ representan el aumento de las pérdidas ocasionadas por una elevación menor de la EB, y vienen dadas por (9) y (10) respectivamente:

$$
L_{b s h}= \begin{cases}-18 \log \left(1+\Delta h_{b}\right) & \text { para } h_{b}>h_{\text {roof }} \\ 0 & \text { para } h_{b} \leq h_{\text {roof }}\end{cases}
$$

En la cual $\Delta h_{\mathrm{b}}$ es la diferencia entre la altura a la que se encuentra la antena de la EB y la altura media de edificios que la rodean (10):

$$
K_{a}=\left\{\begin{array}{rrr}
54 & \text { para }_{b}>h_{\text {roof }} \\
54-0.8 \Delta h_{b} & \text { parad } 0.5 \mathrm{kmy} h_{b} \leq h_{\text {roof }} \\
54-1.6 \Delta h_{b} d \text { parad } 0.5 y h_{b} \leq h_{\text {roof }}
\end{array}\right.
$$

Los términos $K_{\mathrm{d}}$ y $K_{\mathrm{f}}$ en (8) se relacionan con la atenuación por difracción en los múltiples semiplanos y están definidos por (11) y (12) respectivamente:

$$
\begin{aligned}
& K_{d}= \begin{cases}18 & \text { para } h_{b}>h_{\text {roof }} \\
18-15 \Delta h_{b} / h_{\text {roof }} & \text { para } h_{b} \leq h_{\text {roof }}\end{cases} \\
& K_{f}=-4+\left\{\begin{array}{l}
0.7\left(\frac{f}{925}-1\right) \text { para áreas suburbanas } \\
1.5\left(\frac{f}{925}-1\right) \text { para áreas úrbanas }
\end{array}\right.
\end{aligned}
$$

\section{Modelo Interino de la Universidad de Stanford (SUI)}

SUI se deriva del modelo Hata. Es válido para alturas, de la EM entre 2 m y 3 m y de la EB entre 10m y 80m. El rango de frecuencia para el modelo va desde los $0 \mathrm{MHz}$ hasta los $2000 \mathrm{MHz}$ [17].

La formulación empírica de este modelo clasificó los escenarios de propagación en tres tipos de terreno $(A, B, C)$ : TIPO A: escenario con las mayores pérdidas donde predomina el terreno montañoso y densidad promedio de vegetación.

TIPO B: se atribuye a un terreno donde las pérdidas son intermedias, de relieve plano y con densidad promedio de árboles.

TIPO C: caracterizado por la menor cantidad de pérdidas, terreno plano y densidad ligera de la vegetación.

Los tipos de escenarios explicados fueron categorizados a partir de la dispersión Doppler, dispersión por retardo y condiciones LOS y NLOS [18].

Las pérdidas del modelo SUI se presentan en (13):

$$
P L(d B)=A+10 \gamma \log \frac{d}{d_{o}}+X_{f}+X_{h}+S
$$

$A$ corresponde a las pérdidas por trayectoria en el espacio libre y viene dada por (14):

$$
A=20 \log _{10}\left(\frac{4 \pi d_{o}}{\lambda}\right)
$$

Donde $d_{\mathrm{o}}$ es una distancia de referencia $(100 \mathrm{~m}), \lambda$ es la longitud de onda y $\Upsilon$ el exponente de pérdidas que está definido por (15):

$$
\gamma=a-b h_{b}+c / h_{b}
$$


En esta ecuación $h_{\mathrm{b}}$ es la altura de la EB, $a, b$ y $c$ son constantes que pueden ser consultados en la Tabla $1 . X f$ y $X h$ son factores de corrección de frecuencia y altura de la EM respectivamente, los cuales vienen dados por (16) y (17):

$$
\begin{gathered}
X_{f}=6 \log \left(\frac{f}{2000}\right) \\
X_{h}=H \log \left(\frac{h_{r}}{2000}\right)
\end{gathered}
$$

$S$ corresponde al factor de desvanecimiento y su valor es presentado en la Tabla 1.

Con el propósito de caracterizar el comportamiento de las pérdidas de propagación, se realizaron mediciones de potencia en una zona de la ciudad de Barranquilla donde predominan edificaciones con más de cuatro pisos de altura, existe densidad media de vegetación y hay alto tráfico vehicular y peatonal. Por lo anterior, y siguiendo la recomendación P.1411-10 de la Unión Internacional de Telecomunicaciones (UIT), la zona considerada en el estudio presenta características que permiten clasificarla como Urbana de construcción alta [19].

TABLA 1.

\begin{tabular}{|c|c|c|c|}
\hline \multirow{2}{*}{ Parámetros del modelo } & \multicolumn{3}{|c|}{ Tipo de terreno } \\
\hline & A & $\mathrm{B}$ & $\mathrm{C}$ \\
\hline $\mathrm{a}$ & 4.6 & 0.0075 & 12.6 \\
\hline $\mathrm{b}$ & 4 & 0.0065 & 17.1 \\
\hline $\mathrm{c}$ & 3.6 & 0.005 & 20 \\
\hline $\mathrm{S}$ & 10.6 & 9,6 & 8,2 \\
\hline $\mathrm{H}$ & 10.8 & 10,8 & 20 \\
\hline
\end{tabular}

Parámetros del modelo Interino de la Universidad de Satnford.

Fuente: [9].

Se obtuvieron los valores de potencia en un drive test, con un receptor incorporado en un automóvil desplazándose aproximadamente a $50 \mathrm{KM} / \mathrm{h}$. Para obtener las pérdidas en cada punto de medición, se utilizó el método empleado en [20], en el cual se despejó la pérdida por trayectoria de (18):

$$
P_{r x}=P_{t x}+G_{t x}+G_{r x}-P L
$$

En la que Prx corresponde a potencia medida en la EM, Ptx es la potencia de transmisión de la EB, Gtx y Grx son las ganacias de las antenas del transmisor y receptor respectivamente.

En la Fig. 1 se observa la ruta seguida y la ubicación de las EB (marcadas con códigos SC) que ofrecen cobertura en la zona donde se realizó el drive test.

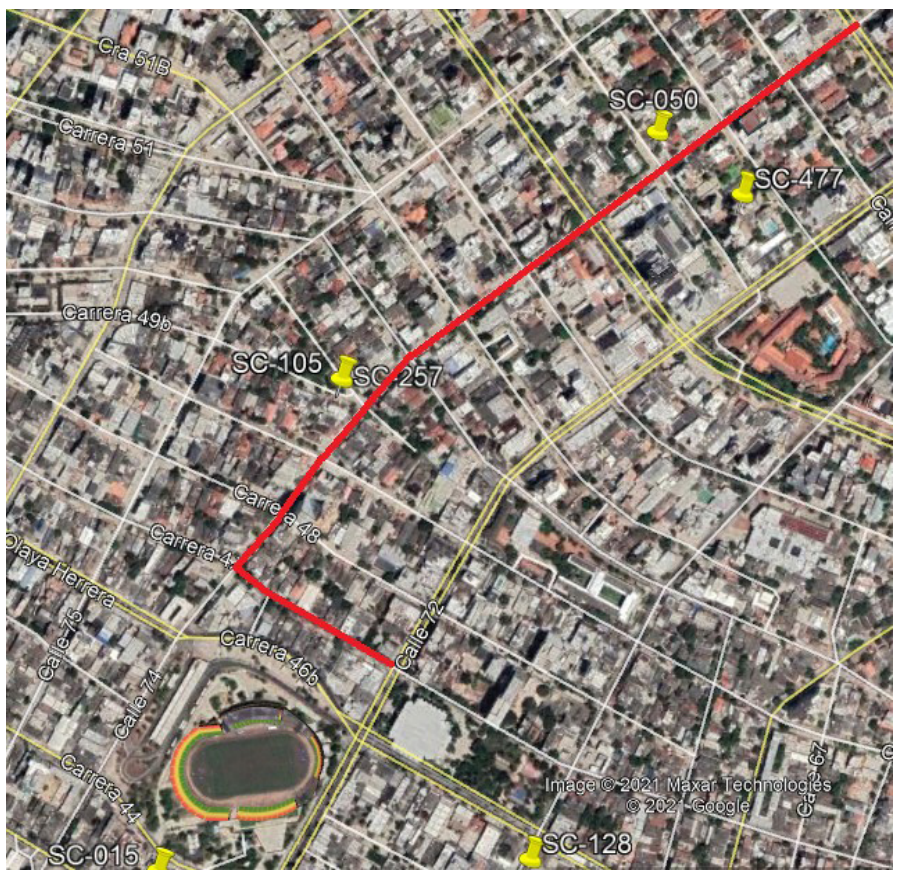

Fig. 1. Ruta de la prueba y ubicación de estaciones base. Fuente: Autores. 
Para obtener los datos de potencia en el enlace descendente, se conectó un teléfono móvil a la herramienta computacional TEMS Investigation, que además permite conocer información de las EB que proporcionan cobertura. Además, cada punto de medición fue referenciado por coordenadas geográficas a través de la conexión con un Equipo de Posicionamiento Global (GPS) del fabricante Royal Tech. Todos los datos correspondientes a la campaña de medición fueron almacenados en un computador portátil. Las mediciones de potencia se hicieron en la banda de los $1900 \mathrm{MHZ}$ y en total fueron 11565 .

En la evaluación de rendimiento de los modelos de propagación se tuvieron en cuenta: la pérdida por trayectoria media, el error de predicción y el error de predicción porcentual; como se hizo en otras investigaciones [8], [21]. El error medio y el error porcentual se obtuvieron por medio de (19) y (20).

$$
\begin{aligned}
& \delta_{\text {error }}=P L_{\text {medida }}-P L_{\text {modelo }} \\
& \delta_{\text {error }}=\left[\left(P L_{\text {medida }}-P L_{\text {modelo }}\right) / P L_{\text {medida }}\right] * 100 \%
\end{aligned}
$$

Donde $P L_{\text {medida }}$ es la pérdida por trayectoria medida, $P L_{\text {modelo }}$ es la pérdida calculada con cada modelo y $\delta e r r o r$ el error de predicción.

\section{Contribuciones y Resultados}

Las pérdidas por trayectoria con cada modelo fueron calculadas en el área de cobertura de siete EB ubicadas en una zona urbana de Barranquilla. En la comparación de los modelos se utilizaron datos obtenidos de la EB de un operador, ubicada en las coordenadas 11.0005 de latitud y -74.801 de longitud, identificada como SC-477. La antena de la EB seleccionada es de referencia K742215 de 18 dBi de ganancia y fue ubicada a 33 metros de altura, con 20 Watts de potencia operando en la banda de $1900 \mathrm{MHz}$. Referente al sistema de recepción, la altura de su antena fue $1.2 \mathrm{~m}$ y su ganancia de $2.2 \mathrm{dBi}$.

Medias de error con tendencia a cero dBs, significa que los modelos representan con precisión las pérdidas por trayectoria en el escenario seleccionado. Un aumento en la media del error muestra que el modelo reduce su eficiencia en la estimación. El análisis de los errores comparativos se presenta en las Tabla 2, Tabla 3 y Tabla 4. En el caso de la evaluación del modelo SUI, se estableció que las condiciones del entorno estudiado se enmarcan en la categoría de terreno $B$.

TABLA 2.

ANÁlisis DEl ERROR PARA El MODELo HATA

\begin{tabular}{|c|l|l|l|c|}
\hline EB & Pérdidas Medidas & Pérdida por trayectoria promedio $(\mathrm{dB})$ & Error de Predicción $(\mathrm{dB})$ & Error relativo (\%) \\
\hline SC- 477 & 140.6 & 233.4 & -92.8 & 66.14 \\
\hline \multicolumn{5}{c}{ Fuente: Autores. } \\
TABLÁLISIS DEL ERROR PARA EL ModeLL WALFISCH-IKEGAMI
\end{tabular}

\begin{tabular}{|c|l|l|l|l|}
\hline EB & Pérdidas Medidas & Pérdida por trayectoria promedio (dB) & Error de Predicción (dB) & Error relativo (\%) \\
\hline SC- 477 & 140.6 & 108.3 & 32.6 & 23.1 \\
\hline
\end{tabular}

Fuente: Autores.

TABla 4.

ANÁLISIS DEL ERROR PARA EL MODELo SUI TIPo B

\begin{tabular}{|c|c|c|c|c|}
\hline EB & Pérdidas Medidas & Pérdida por trayectoria promedio $(\mathrm{dB})$ & Error de Predicción $(\mathrm{dB})$ & Error relativo (\%) \\
\hline SC-477 & 140.6 & 179.3 & -8.7 & 27.7 \\
\hline
\end{tabular}

Fuente: Autores.

Los elevados valores de error muestran que los modelos escogidos se alejan en un alto porcentaje de las mediciones hechas en el escenario estudiado. El modelo con mejor estimación de las pérdidas es el WalfischIkegami con error relativo promedio de $23.1 \%$ tal cual se observa en la Tabla 3, siendo menor a lo obtenido con los modelos COST-231 Hata y SUI tipo B. Sin embargo, el alto nivel de error indica una baja estimación de las pérdidas y por tanto un bajo rendimiento de cada uno de los modelos. Con el modelo Walfisch-Ikegami 
solo el $9.85 \%$ de valores calculados tienen error menor a $28 \mathrm{~dB}$ y el $77.7 \%$ se encuentran entre $28 \mathrm{~dB}$ a 36 dB. En ningún caso se presentó un error menor a $20 \mathrm{~dB}$. El modelo con error medio más alto fue el COST231 Hata, tal como se presenta en la Tabla 2.

\section{Conclusiones}

Tres modelos de propagación empíricos (COST-231 Hata, Walfisch-Ikegami y SUI) fueron evaluados para determinar su efectividad en la estimación de pérdidas por trayectoria en un sector categorizado como urbano de la ciudad de Barranquilla. Los modelos considerados no presentaron una buena estimación de las pérdidas debido a los altos valores de error. Por lo anterior, se hace necesario el ajuste de estos modelos con el fin de calibrarlos adecuadamente y mejorar la estimación de las pérdidas. El modelo Walfisch-Ikegami fue el más preciso, con valores de error relativo menores a los del SUI tipo B y el COST 231-Hata, siendo este último el del peor resultado.

\section{Trabajos Futuros}

Entre las recomendaciones de trabajos futuros, y con el objetivo de ampliar los resultados, se recomienda extender la campaña de medición a otras áreas urbanas de la ciudad de Barranquilla. Además, resulta importante la consideración de otros modelos de propagación con el fin de ampliar la validación aquí presentada.

\section{REFERENCIAS}

[1] T. S. Rappaport, Wireless Communications: Principles and practice, 2 ed. NJ, USA: Prentice Hall, 2002.

[2] N. Blaunstein \& C. Christodoulou, Radio propagation and adaptative antennas for wireless communications. NJ, USA: Wiley, 2007.

[3] S. M. Tasmeeh Ahsan, F. Jahan \& N. M. Proma, "Inspection of picocell's performance using different models in different regions," presente at 5th Int Conf Comput Commun Syst, ICCCS 2020, SHG, CN, pp. 891-894, 2020. http://oi.org/10.1109/ ICCCS49078.2020.9118503

[4] COST telecomunications, COST Action 231. Digital mobile radio towards future generation systems, BRU, BE: EC, Final report, EUR 18957, 1999. Available: https://op.europa.eu/en/publication-detail/-/publication/f2f42003-4028-4496-af95-beaa$38 \mathrm{fd} 475 \mathrm{f}$

[5] R. Saidi, N. Cherrid \& T. Bentahar, "Study of the Prediction of Way Weakening in Mobile Radio Service: Applied to a Part of the City of Batna-Algeria," presented at Int. Conf. Adv. Syst. Emergent Technol, IC_ASET 2020, Hammamet, TUN, pp. 389-393, 15-18 Dec 2020. http://doi.org/10.1109/IC_ASET49463.2020.9318308

[6] R. Drozdova \& V. Akhpashev, "Ordinay Least Squares in COST 231 HATA Key parameters optimization base on experimental data," in 2017 International Multi-Conference on Engineering, Computer and Information Sciences, SIBIRCON, NOV, RU, pp. 21-23, 18-22 Sept 2017. http://doi.org/10.1109/SIBIRCON.2017.8109878

[7] H. S. Jo, C. Park, E. Lee, H. K. Choi \& J. Park, "Path loss prediction based on machine learning techniques: Principal component analysis, artificial neural network and gaussian process," Sensors (Switzerland), vol. 20, no. 7, pp. 1-23, 2020. http:// doi.org/10.3390/s20071927

[8] A. Barrios, R. Arjona \& R. Álvarez, "Comparación de modelos de radiopropagación en el área suburbana de la ciudad de Barranquilla," Rev Colomb Tecnol Av, vol. 2, no. 32, pp. 78-85, 2018. Disponible en http://revistas.unipamplona.edu.co/ojs_viceinves/index.php/RCTA/article/view/3029

[9] UIT-R, "Datos de propagación y métodos de predicción para la planificación de los sistemas de radiocomunicaciones de exteriores de corto alcance y redes de radiocomunicaciones de área local en la gama de frecuencias de $300 \mathrm{MHz}$ a $100 \mathrm{GHz}$ Serie P," Geneva, Switzerland: ITU, P.1411-7, 2013. Available: https://www.itu.int/rec/R-REC-P.1411-7-201309-S/en

[10] S. Mohanty \& S. Mishra, "Performance evaluation of wireless propagation models for long term evolution using NS-3," presente at 2015 Int Conf Man Mach Interfacing, MAMI, BBSR, IMD, 17-19 Dec. 2016. http://doi.org/10.1109/MAMI.2015.7456599

[11] W. Bhupuak \& S. Tooprakai, "Path loss comparison in $850 \mathrm{MHz}$ and $1800 \mathrm{MHz}$ frequency bands," presented at 13th Int Conf Electr Eng Comput Telecommun Inf Technol, ECTI-CON, CNX, TH, 28 Jun.-1 Jul 2016. http://doi.org/10.1109/ECTICon.2016.7561295

[12] H. Xu, C. Shi, W. Zhang \& Y. Yang, "Field testing, modeling and comparison of multi frequency band propagation characteristics for cellular networks," presented at 2016 IEEE Int Conf Commun, ICC 2016, KUL, MY, 22-27 May 2016. http://doi. org/10.1109/ICC.2016.7510961

[13] T. Acar, F. Caliskan \& E. Aydin, "Comparison of computer-based propagation models with experimental data collected in an urban area at $1800 \mathrm{MHz}$," presented at 2015 IEEE 16th Annu Wirel Microw Technol Conf, WAMICON, CB, FL, USA, 13-15 Apr 2015. http://doi.org/10.1109/WAMICON.2015.7120381

[14] N. Belhadj, B. Oueslati \& T. Aguili, "Adjustment of Cost231 Walfisch-Ikegami model for HSPA+ in Tunisian urban environments," presented at 2nd World Symposium on Web Applications and Networking, WSWAN, DTTZ, TN, 21-23 Mar 2015. http://doi.org/10.1109/WSWAN.2015.7210330

[15] L. Schirru, M. B. Lodi, A. Fanti \& G. Mazzarella, "Improved COST 231-WI Model for Irregular Built-Up Areas 2 Modified Version of the Cost 231 Walfisch-Ikegami Model 1 Introduction 4 Results 3 Measurement Campaign," presented at XXXIV General Assembly and Scientific Symposium (GASS) of the International Union of Radio Science, URSI GASS 2020, ROM, IT, 29 Aug-5 Sept 2020. Available from https://www.ursi.org/proceedings/procGA20/papers/Schirruetal.pdf

[16] V. S. Anusha, G. K. Nithya \& S. N. Rao, "A comprehensive survey of electromagnetic propagation models," presented at 2017 IEEE Int Conf Commun Signal Process, ICCSP, MÄS, IN, 6-8 Apr 2017. http://doi.org/10.1109/ICCSP.2017.8286627 
[17] P. K. Sharma, D. Sharma \& T. V. Sai, "Optimization of propagation path loss model in 4G wireless communication systems," presented at 2nd Int Conf Inven Syst Control, ICISC, CJB, IN, 19-20 Jan 2018. http://doi.org/10.1109/ICISC.2018.8399004

[18] A. Mahmood, S. Khan, S. Hussain \& M. Zeeshan, "Performance Analysis of Multi-User Downlink PD-NOMA under sui Fading Channel Models," IEEE Access, vol. 9, pp. 52851-52859, Mar 2021. http://doi.org/10.1109/ACCESS.2021.3070147

[19] UIT-R, Datos de propagación y métodos de predicción para la planificación de los sistemas de radiocomunicaciones de exteriores de corto alcance y redes de radiocomunicaciones de área local en la gama de frecuencias de $300 \mathrm{MHz}$ a $100 \mathrm{GHz}$ Serie P, Geneva, Switzerland: ITU, P.1411-10, 2019. Available: https://www.itu.int/rec/R-REC-P.1411-10-201908-I/es

[20] C. Phillips, D. Sicker \& D. Grunwald, "A survey of wireless path loss prediction and coverage mapping methods," IEEE Commun Surv Tutorials, vol. 15, no. 1, pp. 255-270, Mar 2012. http://doi.org/10.1109/SURV.2012.022412.00172

[21] A. Bhuvaneshwari \& T. Sathyasavithri, "Comparative analysis of mobile radio path loss models for suburban environment in Southern India," presented at 2013 Int Conf Emerg Trends VLSI Embed Syst Nano Electron Telecommun Syst, ICEVENT, TVM, IN, 7-9 Jan 2013. http://doi.org/10.1109/ICEVENT.2013.6496544

Alexis Barrios-Ulloa es Ingeniero Electrónico y Telecomunicaciones de la Universidad Autónoma del Caribe (Colombia) y Magister en Ingeniería de la Universidad Tecnológica de Bolívar (Colombia). Es profesor del departamento de Ingeniería Electrónica de la Universidad de Sucre (Colombia). Actualmente se encuentra desarrollando un Doctorado en Tecnologías de la Información y la Comunicación en la Universidad de la Costa (Colombia) gracias a una beca del Ministerio de Ciencia Tecnología e Innovación de Colombia. Sus áreas de interés son las comunicaciones inalámbricas, específicamente el modelamiento de la propagación de ondas de radio. 